\title{
ChemComm
}

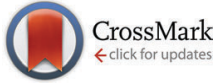

Cite this: Chem. Commun., 2016, 52,6926

Received 15th February 2016, Accepted 28th April 2016

DOI: $10.1039 / c 6 c c 01407 k$

www.rsc.org/chemcomm

\section{One-step growth of lanthanoid metal-organic framework (MOF) films under solvothermal conditions for temperature sensing $\dagger$}

\author{
Xue Liu, Wentian Fu and Elisabeth Bouwman*
}

\begin{abstract}
A one-step direct solvothermal synthesis of an Ln metal-organic framework (MOF) film is reported. The $\mathrm{LnHL}$ ( $\mathrm{Ln}=\mathrm{Tb}$ and $\mathrm{Gd}$ ) films that were deposited on $\mathrm{a} \mathrm{Gd}_{2} \mathrm{O}_{3}$ subtrate are continuous and smooth. The $\mathrm{Gd}_{0.9} \mathrm{~Tb}_{0.1} \mathrm{HL}$ film can be used as a ratiometric thermometer, showing good linear behaviour in the temperature range of $110-250 \mathrm{~K}$ with a sensitivity up to $0.8 \% \mathrm{~K}^{-1}$.
\end{abstract}

Metal-organic frameworks (MOFs) are promising materials for many applications such as gas storage and separation, ion and small molecule sensing, catalysis and proton conduction. ${ }^{1-4}$ Lanthanoid MOFs are especially interesting materials due to their unique luminescence properties like high quantum yields, characteristic sharp line emissions and long lifetime. ${ }^{5,6}$

Recently, the preparation and study of films of MOFs on supports gained increasing attention. ${ }^{7-11}$ Due to the brittleness and insolubility of MOF crystals, thin films of such materials are difficult to process with common surface deposition methods, limiting the application of MOF films. ${ }^{12}$ Five major methods to fabricate MOF films have been reported: (1) deposition of MOF films directly onto bare substrates or functionalized substrates with organic molecules; (2) deposition of MOF films on seeded substrates; (3) deposition of preformed MOF nanocrystals; (4) layer-by-layer deposition of MOF films; (5) electrodeposition. ${ }^{13,14}$ Gold, silicon wafers, graphene, glass, indium tin oxide (ITO), and porous aluminium oxide are mostly used as substrates for MOF films. ${ }^{13-20}$

Until now, most research concerning MOF films focuses on transition metals like $\mathrm{Zn}, \mathrm{Cu}, \mathrm{Mn}$ and Fe. Only few lanthanoid MOF films have been reported and most of these have been synthesized by post-functionalization strategies based on the 'parent' zinc, copper or indium MOF films, or by spin coating or dip coating of Ln MOF nanocrystals. ${ }^{7,21-30}$ Meyer et. al. reported the in situ growth of $\mathrm{Sr} / \mathrm{Eu}$ MOFs on anodic aluminum oxide (AAO) membranes using a melt approach with $\mathrm{Eu}$ and $\mathrm{Sr}$ metals and

Leiden Institute of Chemistry, Leiden University, P.O. Box 9502, 2300 RA Leiden, The Netherlands. E-mail: bouwman@chem.leidenuniv.nl

$\dagger$ Electronic supplementary information (ESI) available: Synthesis procedure, excitation and emission spectra, SEM, PXRD. See DOI: 10.1039/c6cc01407k imidazole. Alternatively, the growth process was carried out by an electride-induced reaction from liquid ammonia. ${ }^{31}$

Temperature is one of the most important parameters both in scientific research and industry. ${ }^{32}$ In recent years, lanthanoidbased ratiometric luminescence thermometers have drawn great attention; the investigations in this field are mainly based on lanthanoid coordination compounds, or $\mathrm{Er}^{\mathrm{III}} / \mathrm{Yb}^{\mathrm{III}}$ nanoparticles. ${ }^{33}$ As the number of combinations of organic ligands with lanthanoid ions is unlimited, the composition and properties of a luminescence thermometer based on Ln MOFs can be varied easily. By careful engineering of a film of such a luminescence thermometer onto a suitable substrate a novel device can be developed with which surface temperature distribution can be easily mapped via a non-invasive method.

It has been reported that $\mathrm{Zn}$ and $\mathrm{Cu}$ MOF films can grow on zinc or copper metal surfaces, and indium-containing MOF films can grow on glass containing an indium tin oxide (ITO) coating. ${ }^{15,34-36}$ As most Ln MOFs are synthesized by hydrothermal or solvothermal methods, a method to deposit Ln MOF films using such hydro/solvothermal conditions would be highly valuable. To the best of our knowledge, such direct hydrothermal or solvothermal synthesis of Ln MOF films has not yet been reported, which may attributed mainly to the lack of a suitable substrate. Herein we report for the first time the use of an Ln oxide as substrate for the deposition of Ln MOF films by in situ solvothermal methods. Potentially, this method can be used for the production of a wide range of Ln MOF films.

Pellets of a $\mathrm{Gd}_{2} \mathrm{O}_{3}$ substrate (diameter $13 \mathrm{~mm}$, thickness around $1.5 \mathrm{~mm}$ ) were prepared by compressing $\mathrm{Gd}_{2} \mathrm{O}_{3}$ powder under static 10 tons pressure for $10 \mathrm{~min}$. These pellets were transferred to a tube furnace and sintered at $1500{ }^{\circ} \mathrm{C}$ for $15 \mathrm{~h}$ to increase their mechanical strength (see $\mathrm{ESI} \dagger$ for detailed description of the preparation). We envisaged that surface Gd ions from the $\mathrm{Gd}_{2} \mathrm{O}_{3}$ substrate could act as nucleation sites for the growth of the MOF and thereby assist in the formation and improve the quality of the Ln MOF. The Gd ${ }^{\mathrm{III}}$ ion does not show any luminescence properties due to its $\mathrm{f}^{7}$ electron configuration, and thus does not interfere with the luminescence properties of 


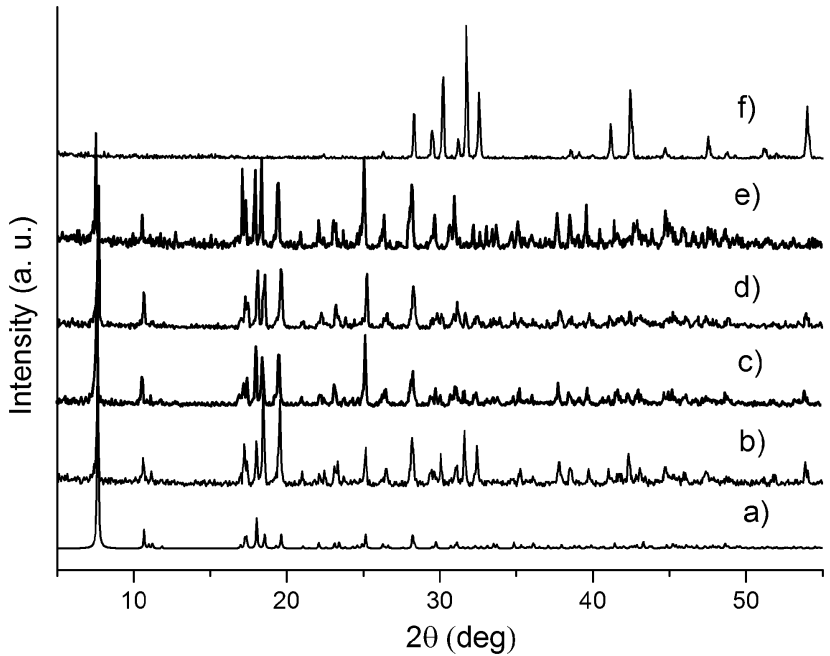

Fig. 1 Simulated PXRD pattern calculated from the single crystal structure of $\mathrm{TbHL}(\mathrm{a})$ and the experimental PXRD patterns of films of $\mathrm{TbHL}(\mathrm{b}), \mathrm{Gd}_{0.9} \mathrm{~Tb}_{0.1} \mathrm{HL}$ (c), $\mathrm{Gd}_{0.9} \mathrm{~Tb}_{0.1} \mathrm{HL}$ after low temperature measurement (d), $\mathrm{GdHL}$ (e) and the $\mathrm{Gd}_{2} \mathrm{O}_{3}$ substrate $(\mathrm{f})$.

the film. Films of the compounds TbHL, GdHL and $\mathrm{Gd}_{0.9} \mathrm{~Tb}_{0.1} \mathrm{HL}$ $\left(\mathrm{H}_{4} \mathrm{~L}=\right.$ 5-hydroxy-1,2,4-benzenetricarboxylic acid) on $\mathrm{Gd}_{2} \mathrm{O}_{3}$ substrates were obtained using the solvothermal method with the same conditions as for the preparation of the bulk crystals. ${ }^{37}$ The films were characterized with powder X-ray diffraction (PXRD) and scanning electron microscopy (SEM). The PXRD patterns of the films are highly similar to the simulated pattern from the single crystal X-ray structure of TbHL reported previously (Fig. 1), indicating that indeed the MOF grown on the $\mathrm{Gd}_{2} \mathrm{O}_{3}$ surface has the same structure as the bulk material. ${ }^{37}$

SEM was used to study the morphology of the $\mathrm{Gd}_{2} \mathrm{O}_{3}$ substrate and the TbHL film. The $\mathrm{Gd}_{2} \mathrm{O}_{3}$ substrate is a porous material (Fig. 2a). The TbHL film is packed tightly and smoothly onto the surface of the $\mathrm{Gd}_{2} \mathrm{O}_{3}$ substrate (Fig. 2b); the thickness of the TbHL film is approximately $10 \mu \mathrm{m}$ (Fig. $2 \mathrm{c}$ and d).

Similar to the growth mechanism of the In MOF film on ITO glass, the $\mathrm{Cu}$ MOF film on a copper grid and the zinc MOF film on activated metallic zinc, we assume that the $\mathrm{Gd}_{2} \mathrm{O}_{3}$ substrate provides $\mathrm{Gd}^{\mathrm{III}}$ ions as nucleation sites to improve the growth of the MOF at its surface. ${ }^{15,34-36}$ In addition the porous structure may provide better adhesion between the MOF film and the substrate. However, the presence of a porous structure by itself is not enough for the formation of a MOF film, as using a porous $\mathrm{Al}_{2} \mathrm{O}_{3}$ pellet as the substrate did not yield the desired films.

Under $366 \mathrm{~nm}$ UV light (high pressure $\mathrm{Hg}$ lamp), the $\mathrm{Gd}_{2} \mathrm{O}_{3}$ substrate is not luminescent while the TbHL film on this substrate exhibits intense green luminescence (Fig. S1, ESI $\dagger$ ). When irradiated with $325 \mathrm{~nm}$ UV light, the TbHL film displays the characteristic $\mathrm{Tb}^{\mathrm{III}}$ sharp-line emissions at $490\left({ }^{5} \mathrm{D}_{4} \rightarrow{ }^{7} \mathrm{~F}_{6}\right)$, $541\left({ }^{5} \mathrm{D}_{4} \rightarrow{ }^{7} \mathrm{~F}_{5}\right)$, $587\left({ }^{5} \mathrm{D}_{4} \rightarrow{ }^{7} \mathrm{~F}_{4}\right)$ and $622 \mathrm{~nm}\left({ }^{5} \mathrm{D}_{4} \rightarrow{ }^{7} \mathrm{~F}_{3}\right)$ (Fig. 3a). The dominant emission belongs to the hypersensitive ${ }^{5} \mathrm{D}_{4} \rightarrow{ }^{7} \mathrm{~F}_{5}$ transition $(541 \mathrm{~nm})$. Emission from the ligand is not observed, indicating efficient energy-transfer from the ligand to the $\mathrm{Tb}^{\mathrm{III}}$ center. A GdHL film on the $\mathrm{Gd}_{2} \mathrm{O}_{3}$ substrate shows a weak broad-band emission from $350-650 \mathrm{~nm}$ as a result of
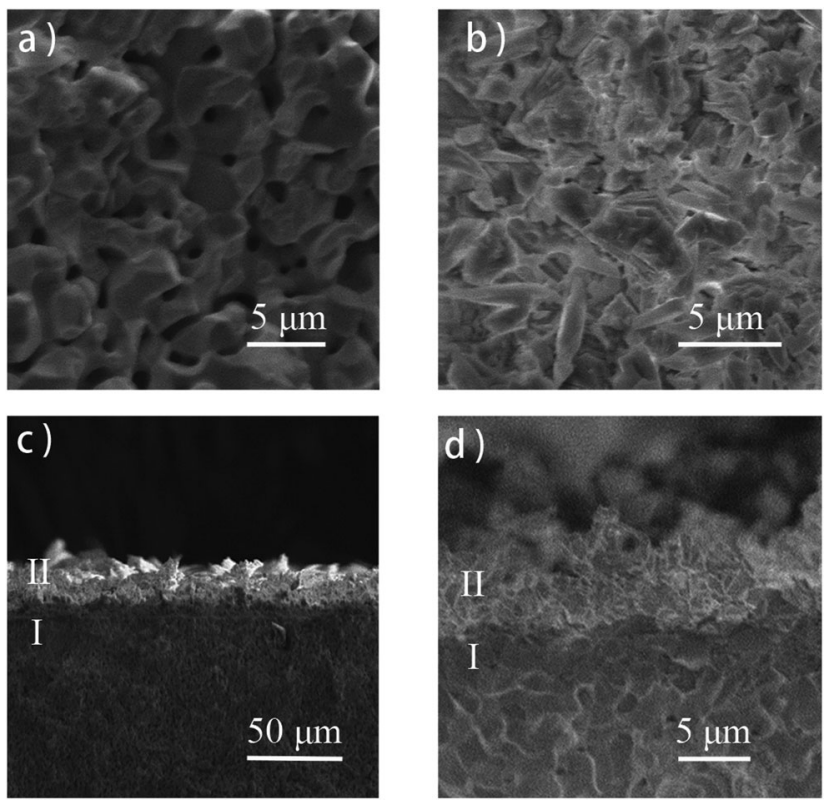

Fig. 2 SEM images of (a) $\mathrm{Gd}_{2} \mathrm{O}_{3}$ substrate; (b) a TbHL film on $\mathrm{Gd}_{2} \mathrm{O}_{3}$; (c) cross-sectional image of the $\mathrm{TbHL}$ film on $\mathrm{Gd}_{2} \mathrm{O}_{3}$; (d) enlarged image of the $\mathrm{TbHL}$ film on $\mathrm{Gd}_{2} \mathrm{O}_{3}$. (I, $\mathrm{Gd}_{2} \mathrm{O}_{3} ; \mathrm{II}, \mathrm{TbHL}$ film).

ligand-centred phosphorescence (Fig. $3 \mathrm{~b}$ ). $\mathrm{A} \mathrm{Gd}_{0.9} \mathrm{~Tb}_{0.1} \mathrm{HL}$ film, doped with $10 \% \mathrm{~Tb}$, clearly shows $\mathrm{Tb}^{\mathrm{III}}$ emission whereas the ligand-based phosphorescence is very weak (Fig. 3c). The excitation spectrum of the $\mathrm{Gd}_{0.9} \mathrm{~Tb}_{0.1} \mathrm{HL}$ film, monitored at $541 \mathrm{~nm}$, reveals a broad excitation band with a maximum at $325 \mathrm{~nm}$ that is ascribed to ligand-centred excitation. The two small peaks in the excitation spectrum at $369\left({ }^{5} \mathrm{~L}_{10} \leftarrow{ }^{7} \mathrm{~F}_{6}\right)$ and $379 \mathrm{~nm}\left({ }^{5} \mathrm{D}_{3} \leftarrow{ }^{7} \mathrm{~F}_{6}\right)$ are a result of the direct excitation of the $\mathrm{Tb}^{\mathrm{III}}$ ion (Fig. S2, ESI $\dagger$ ). The emission lifetimes of $\mathrm{Tb}$ emission in the TbHL film and $\mathrm{Gd}_{0.9} \mathrm{~Tb}_{0.1} \mathrm{HL}$ film were determined by monitoring the $541 \mathrm{~nm}$ emission. The lifetime of terbium emission in the TbHL film is $0.60 \mathrm{~ms}$, which is shorter than that in the $\mathrm{Gd}_{0.9} \mathrm{~Tb}_{0.1} \mathrm{HL}$ film (0.69 ms) (Fig. S3, ESI $\dagger$ ). Due to short $\mathrm{Tb}-\mathrm{Tb}$ distances $(4.212 \AA$ and $4.532 \AA)$ in the TbHL film, the energy at the emitting level of the $\mathrm{Tb}$ ion can migrate efficiently, increasing the probability of quenching at a defect site. In contrast, in the 'diluted' $\mathrm{Gd}_{0.9} \mathrm{~Tb}_{0.1} \mathrm{HL}$ film the $\mathrm{Gd}^{\mathrm{III}}$ ions effectively act as insulators, preventing energy migration between $\mathrm{Tb}$ ions and thus enhancing the lifetime of $\mathrm{Tb}$ emission.

To evaluate the potential use of the $\mathrm{Gd}_{0.9} \mathrm{~Tb}_{0.1} \mathrm{HL}$ film as a ratiometric thermometer, temperature-dependent emission spectra were recorded. As the temperature increases from 110 to $270 \mathrm{~K}$, the intensity of the $\mathrm{Tb}^{\mathrm{III}}$-based emission changes only slightly. However, the intensity of the ligand-based phosphorescence decreases dramatically with increasing temperature due to the thermal activation of non-radiative decay pathways (Fig. 4a and Fig. S4, ESI $\dagger$ ). The different responses of the two kinds of emission to changes in temperature make this material a good candidate for ratiometric temperature sensing.

We define the thermometer parameter $y$ as the ratio between the intensities $I_{541 \mathrm{~nm}}\left(\mathrm{~Tb},{ }^{5} \mathrm{D}_{4} \rightarrow{ }^{7} \mathrm{~F}_{5}, 541 \mathrm{~nm}\right)$ and $I_{(\mathrm{phos}+\mathrm{Tb})}$ (the total emission intensity from $350-650 \mathrm{~nm}$ ). A good linear 
a)

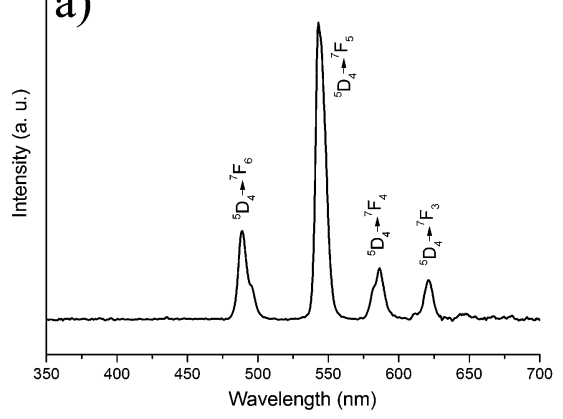

b)

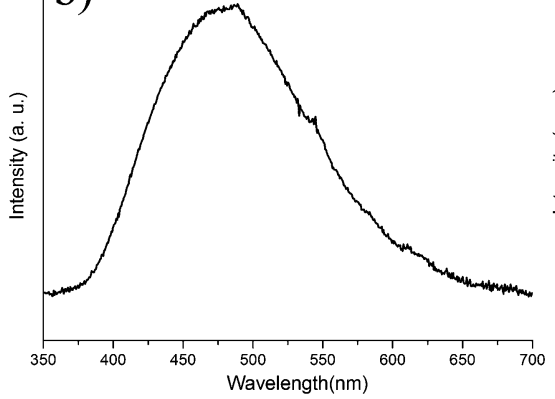

c)

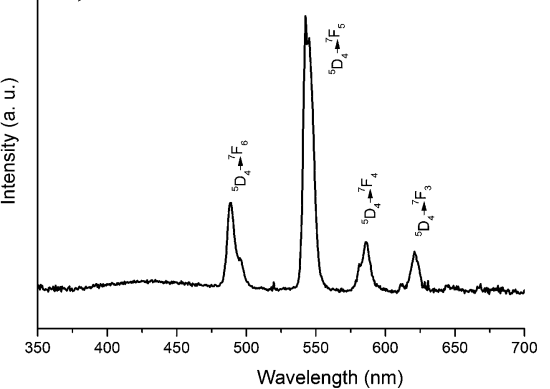

Fig. 3 Room temperature emission spectra of (a) TbHL film; (b) GdHL film; (c) $\mathrm{Gd}_{0.9} \mathrm{~Tb}_{0.1} \mathrm{HL}$ film. ( $\lambda_{\text {exc }}=325 \mathrm{~nm}$ ).

relationship was found in the temperature range of 110-250 K according to eqn (1), with a correlation coefficient $R^{2}=0.994$ (Fig. 4b).

$$
y=0.02481+0.00178 T 110 \mathrm{~K}<T<250 \mathrm{~K}
$$

The thermometer performance is evaluated by the relative sensitivity $\left(S_{\mathrm{r}}\right)$, defined by eqn (2).

$$
S_{\mathrm{r}}=\frac{\mathrm{d} y}{y \mathrm{~d} T}
$$
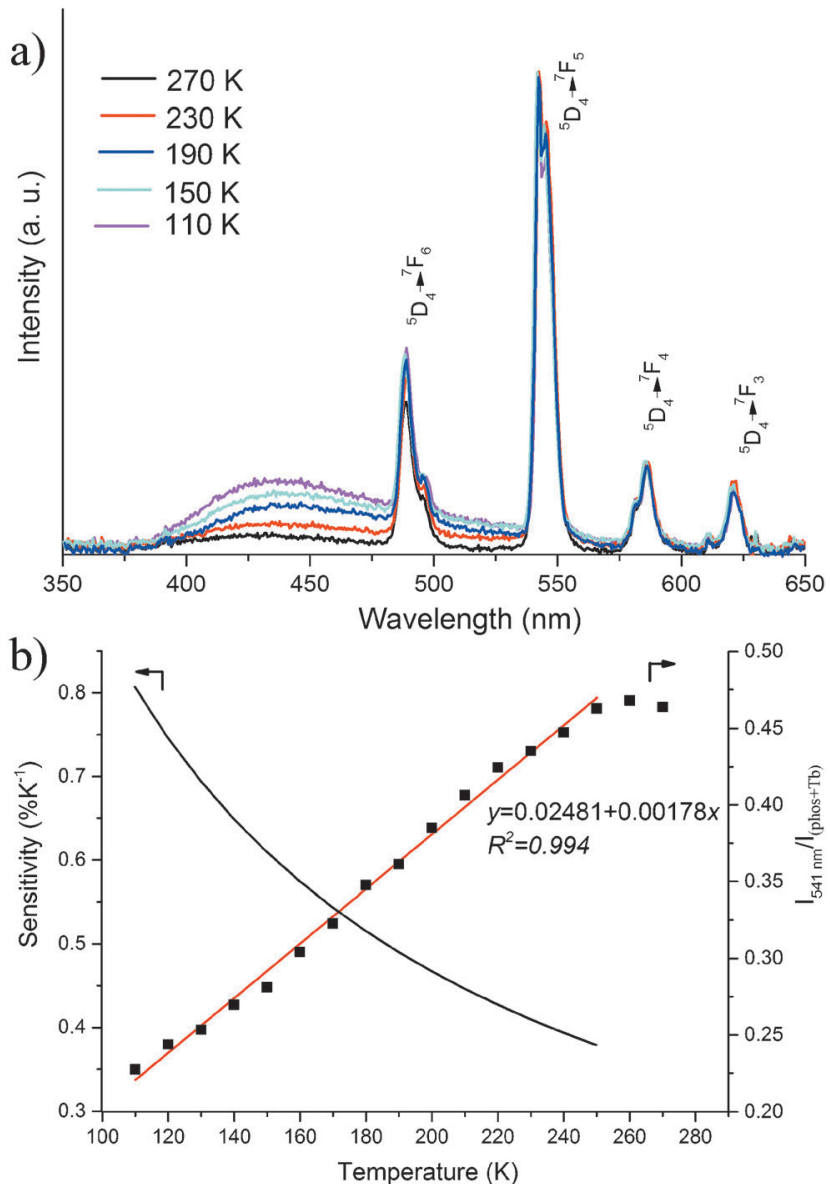

Fig. 4 (a) Emission spectra of $\mathrm{Gd}_{0.9} \mathrm{~Tb}_{0.1} \mathrm{HL}$ films recorded in the temperature range 90-270 K; (b) the emission intensity ratio of $I_{541 \mathrm{~nm}}\left(\mathrm{~Tb},{ }^{5} \mathrm{D}_{4} \rightarrow{ }^{7} \mathrm{~F}_{5}, 541 \mathrm{~nm}\right)$ and $l_{\text {(phos }+\mathrm{Tb})}$ (the emission intensity from $350 \mathrm{~nm}$ to $650 \mathrm{~nm}$ ) for $\mathrm{Gd}_{0.9} \mathrm{~Tb}_{0.1} \mathrm{HL}$ film as a function of temperature (black squares, right axis) with the fitting curve (red line; $R^{2}=0.994$ ) and the relative sensitivity curve (black line, left axis).
The relative sensitivity of our thermometer film is up to $0.8 \% \mathrm{~K}^{-1}$ at $110 \mathrm{~K}$ (Fig. 4b). A comparison of the sensitivity of the ratiometric thermometer in this work with other reported materials is given in Fig. S5 (ESI $\dagger$ ).

The accuracy and precision are other two important parameters to evaluate the performance of a thermometer. The accuracy is related to the correlation coefficient of the fitting curve. The standard deviation of the calibration curve is 0.0057 and the temperature precision is $\pm 3.2 \mathrm{~K}$ based on eqn (1). The luminescence emission colour change of the $\mathrm{Gd}_{0.9} \mathrm{~Tb}_{0.1} \mathrm{HL}$ film from 110 to $250 \mathrm{~K}$ has been visualized in a CIE (Commission International d'Eclairage) chromaticity diagram (Fig. S6, ESI $\dagger$ ). The CIE coordinates at different temperatures as calculated from the emission spectra are provided in Table S1 (ESI $\dagger$ ). The emission colour changes from blue-green at $110 \mathrm{~K}$ to green at $250 \mathrm{~K}$.

The stability of the film was tested by exposing the sample to temperature cycles from 110 to $250 \mathrm{~K}$ for four times which proved it to be rather robust (Fig. S7, ESI $\dagger$ ). After the low-temperature luminescence measurements, PXRD was measured again to confirm the stability of the crystal phase (Fig. 1).

As stated in the introduction, the composition and properties of a luminescence thermometer based on Ln MOFs can be varied easily. As a proof of principle another MOF film was prepared; it appeared that lowering the $\mathrm{Tb}$ content to approximately $1 \%\left(\mathrm{Gd}_{0.99} \mathrm{~Tb}_{0.01} \mathrm{HL}\right)$ resulted in further improvement of the performance (Fig. S8 and S9, ESI $\dagger$ ). The temperature relationship can be fitted with an exponential curve, with a relative sensitivity of $1.7 \% \mathrm{~K}^{-1}$ at $240 \mathrm{~K}$ up to $4.4 \% \mathrm{~K}^{-1}$ at $110 \mathrm{~K}$. It thus appears that with a higher content of $\mathrm{Gd}$ the material becomes more sensitive, however, an even lower concentration of $\mathrm{Tb}$ will make the $\mathrm{Tb}$ emission too weak which will result in increased errors in the measurements.

In summary, for the first time lanthanoid MOF films were synthesized by a direct solvothermal method onto a $\operatorname{Gd}_{2} \mathrm{O}_{3}$ substrate. Surface $\mathrm{Gd}$ ions from the $\mathrm{Gd}_{2} \mathrm{O}_{3}$ substrate act as nucleation sites for the growth of the MOF and thereby assist in the formation and improve the quality of the Ln MOF. This new method unlocks a promising route to the synthesis of a wide range of Ln MOF films. The $\mathrm{Gd}_{0.9} \mathrm{~Tb}_{0.1} \mathrm{HL}$ film was shown to be a potential thermometer for use in the temperature range of 110 to $250 \mathrm{~K}$, with a relative sensitivity up to $0.8 \% \mathrm{~K}^{-1}$, whereas the compound $\mathrm{Gd}_{0.99} \mathrm{~Tb}_{0.01} \mathrm{HL}$ with a lower $\mathrm{Tb}$ content resulted in a relative sensitivity up to $4.4 \% \mathrm{~K}^{-1}$ at $110 \mathrm{~K}$. 
X. Liu gratefully acknowledges a grant from the Chinese Scholarship Council (no. 201206250014). We are grateful to Dr Hadi Arjmandi-Tash for his assistance with the SEM measurements. We thank Dr Hong Zhang and Dr Pengtao Jing (University of Amsterdam) for lifetime measurements.

\section{References}

1 Z. C. Hu, B. J. Deibert and J. Li, Chem. Soc. Rev., 2014, 43, $5815-5840$.

2 X. D. Wang, O. S. Wolfbeis and R. J. Meier, Chem. Soc. Rev., 2013, 42, 7834-7869.

3 M. Gustafsson, A. Bartoszewicz, B. Martín-Matute, J. L. Sun, J. Grins, T. Zhao, Z. Y. Li, G. S. Zhu and X. D. Zou, Chem. Mater., 2010, 22, 3316-3322.

4 Y. Zhu, Y. M. Wang, P. Liu, C. K. Xia, Y. L. Wu, X. Q. Lu and J. M. Xie, Dalton Trans., 2015, 44, 1955-1961.

5 Y. J. Cui, Y. F. Yue, G. D. Qian and B. L. Chen, Chem. Rev., 2012, 112, 1126-1162.

6 J. C. Bunzli and C. Piguet, Chem. Soc. Rev., 2005, 34, 1048-1077.

7 Z. S. Dou, J. C. Yu, Y. J. Cui, Y. Yang, Z. Y. Wang, D. R. Yang and G. D. Qian, J. Am. Chem. Soc., 2014, 136, 5527-5530.

8 Y. S. Li, F. Y. Liang, H. Bux, A. Feldhoff, W. S. Yang and J. Caro, Angew. Chem., Int. Ed., 2010, 49, 548-551.

9 Y. M. Zhu, C. H. Zeng, T. S. Chu, H. M. Wang, Y. Y. Yang, Y. X. Tong, C. Y. Su and W. T. Wong, J. Mater. Chem. A, 2013, 1, 11312-11319.

10 Q. Liu, N. Y. Wang, J. Caro and A. S. Huang, J. Am. Chem. Soc., 2013, 135, 17679-17682.

11 W. J. Li, S. Y. Gao, T. F. Liu, L. W. Han, Z. J. Lin and R. Cao, Langmuir, 2013, 29, 8657-8664.

12 H. P. Liu, H. M. Wang, T. S. Chu, M. H. Yu and Y. Y. Yang, J. Mater. Chem. C, 2014, 2, 8683-8690.

13 D. Zacher, O. Shekhah, C. Wöll and R. A. Fischer, Chem. Soc. Rev., 2009, 38, 1418-1429.

14 X. J. Zhang, W. J. Wang, Z. J. Hu, G. N. Wang and K. Uvdal, Coord. Chem. Rev., 2015, 284, 206-235.
15 Z. S. Dou, J. C. Yu, H. Xu, Y. J. Cui, Y. Yang and G. D. Qian, Thin Solid Films, 2013, 544, 296-300.

16 R. A. Fischer and C. Wöll, Angew. Chem., Int. Ed., 2009, 48, 6205-6208.

17 M. T. Conato and A. J. Jacobson, Microporous Mesoporous Mater., 2013, 175, 107-115.

18 Y. y. Mao, W. Cao, J. W. Li, L. W. Sun and X. S. Peng, Chem. - Eur. J., 2013, 19, 11883-11886.

19 M. C. So, S. Y. Jin, H. J. Son, G. P. Wiederrecht, O. K. Farha and J. T. Hupp, J. Am. Chem. Soc., 2013, 135, 15698-15701.

20 J. F. Yao and H. T. Wang, Chem. Soc. Rev., 2014, 43, 4470-4493.

21 Y. Zhou and B. Yan, J. Mater. Chem. C, 2015, 3, 8413-8418.

22 Y. Lu and B. Yan, J. Mater. Chem. C, 2014, 2, 5526-5532.

23 X. Shen and B. Yan, J. Colloid Interface Sci., 2015, 451, 63-68.

24 C. Liu and B. Yan, Eur. J. Inorg. Chem., 2015, 279-287.

25 Y. Lu and B. Yan, J. Mater. Chem. C, 2014, 2, 7411-7416.

26 H. C. Streit, M. Adlung, O. Shekhah, X. Stammer, H. K. Arslan, O. Zybaylo, T. Ladnorg, H. Gliemann, M. Franzreb, C. Wöll and C. Wickleder, ChemPhysChem, 2012, 13, 2699-2702.

27 H. L. Guo, Y. Z. Zhu, S. L. Qiu, J. A. Lercher and H. J. Zhang, Adv. Mater., 2010, 22, 4190-4192.

28 Z. S. Dou, J. C. Yu, H. Xu, Y. J. Cui, Y. Yang and G. D. Qian, Microporous Mesoporous Mater., 2013, 179, 198-204.

29 T. W. Duan and B. Yan, J. Mater. Chem. C, 2014, 2, 5098-5104.

30 J. Xu, L. Jia, N. Z. Jin, Y. F. Ma, X. Liu, W. Y. Wu, W. S. Liu, Y. Tang and F. Zhou, Chem. - Eur. J., 2013, 19, 4556-4562.

31 L. V. Meyer, J. Vogt, F. A. Brede, H. Schafer, M. Steinhart and K. Muller-Buschbaum, CrystEngComm, 2013, 15, 9382-9386.

32 J. Seyed-Yagoobi, Rev. Sci. Instrum., 1991, 62, 249-250.

33 C. D. S. Brites, P. P. Lima, N. J. O. Silva, A. Millan, V. S. Amaral, F. Palacio and L. D. Carlos, New J. Chem., 2011, 35, 1177-1183.

34 X. Q. Zou, G. S. Zhu, I. J. Hewitt, F. X. Sun and S. L. Qiu, Dalton Trans., 2009, 3009-3013.

35 H. L. Guo, G. S. Zhu, I. J. Hewitt and S. L. Qiu, J. Am. Chem. Soc., 2009, 131, 1646-1647.

36 J. Liu, F. X. Sun, F. Zhang, Z. Wang, R. Zhang, C. Wang and S. L. Qiu, J. Mater. Chem., 2011, 21, 3775-3778.

37 X. Liu, S. Akerboom, M. d. Jong, I. Mutikainen, S. Tanase, A. Meijerink and E. Bouwman, Inorg. Chem., 2015, 54, 11323-11329. 